\title{
THE CLASSIFICATION OF SOLENOIDS
}

\author{
J. M. AARTS AND R. J. FOKKINK
}

(Communicated by James E. West)

\begin{abstract}
An elementary proof is presented of a classification theorem for solenoids.
\end{abstract}

\section{INTRODUCTION}

A solenoid may be defined as the intersection of a sequence of tori $T_{1}, T_{2}, \ldots$ such that $T_{i+1}$ is wrapped around inside $T_{i}$ longitudinally $n_{i}$ times in a smooth fashion without folding back. There is no loss of generality in assuming that all $n_{i}$ are prime numbers greater than one. A solenoid is denoted by $S_{P}$, where $P=\left(p_{1}, p_{2}, \ldots\right)$ is a sequence of prime numbers.

We call the sequences $P$ and $Q$ of primes equivalent and write $P \sim Q$ if a finite number of primes can be deleted from each sequence so that every prime number occurs the same number of times in the deleted sequences. The following classification theorem was conjectured by Bing [3], who noticed the correctness of the "if" part.

Theorem. The solenoids $S_{P}$ and $S_{Q}$ are homeomorphic iff $P \sim Q$.

The "only if" part was proved by McCord [5]. The proof exploits the fact that a solenoid can be endowed with the structure of a topological group. The continuity of the Čech cohomology and Pontrjagin duality are invoked. The aim of this paper is to present a rather elementary proof of the theorem.

\section{MAPPING TORUS}

In $[6, p .392]$ a quite elaborate description of the flow structure of a solenoid is presented. With the above definition of a solenoid, its flow structure may be captured as follows. The transverse (or cross) section of a solenoid is a Cantor set $C$. By following the arc components (or composants) in one of the two possible directions, in a natural way there is defined a return map $\sigma: C \rightarrow C$. The map $\sigma$ essentially is the Poincare return map. The solenoid then may be seen as the mapping torus (or suspension) $\sum(C, \sigma)$, which is obtained from the product $C \times[0,1]$ by identifying each $(x, 1)$ with $(\sigma(x), 0), x \in X$. By

Received by the editors April 8, 1989 and, in revised form, March 6, 1990.

1980 Mathematics Subject Classification (1985 Revision). Primary 54H20; Secondary 54F20. 
regarding $t \in[0,1]$ as time, a transparent picture of the flow structure of the solenoid emerges.

We might as well have started out by defining, for a sequence of primes $P=$ $\left(p_{1}, p_{2}, \ldots\right)$, the solenoid $S_{P}$ as the mapping torus $\sum\left(C_{P}, \sigma\right)$. Here $C_{P}$ is the Cantor set represented as the topological product $C_{P}=\prod_{i=1}^{\infty}\left\{0,1, \ldots, p_{i}-1\right\}$ of the finite discrete spaces $\left\{0,1, \ldots, p_{i}-1\right\}, i=1,2, \ldots$.

The return map $\sigma$ has the form

$$
\begin{aligned}
& \sigma\left(x_{1}, x_{2}, \ldots\right)=\left(x_{1}+1, x_{2}, x_{3}, \ldots\right) \text { if } x_{1}<p_{1}-1, \\
& \sigma\left(p_{1}-1, \ldots, p_{k-1}-1, x_{k}, \ldots\right)=\left(0,0, \ldots, 0, x_{k}+1, \ldots\right) \text { if } x_{k}<p_{k}-1, \\
& \sigma\left(p_{1}-1, p_{2}-1, \ldots\right)=(0,0, \ldots) .
\end{aligned}
$$

For convenience we shall write $\bar{p}=\{0,1, \ldots, p-1\}$ for any number $p \geq$ 1. Thus $C_{P}=\prod_{i=1}^{\infty} \bar{p}_{i}$. The cascade $\left(C_{P}, \sigma\right)$ is sometimes called an adding machine. Actually $C_{P}$ is the topological group of $P$-adic integers, and the return map corresponds to the addition of $(1,0,0, \ldots)$ [4].

The representation of the solenoid $S_{P}=\sum\left(C_{P}, \sigma\right)$ as a mapping torus is by no means unique. If $U$ is any clopen subset of $C_{P}$, then the return map $\sigma_{U}$ can be defined in the same way as above. The map $\sigma_{U}: U \rightarrow U$ can be seen to be a homeomorphism and we find $S_{P}=\sum\left(U, \sigma_{U}\right)$.

There is a simple relation between $\sigma$ and $\sigma_{U}$. For each $x \in U$, let $n(x)$ be the least integer $\geq 1$ such that $\sigma^{n(x)}(x) \in U$. Then $\sigma_{U}(x)=\sigma^{n(x)}(x)$. This motivates the following definition.

Definition. Let $P=\left(p_{1}, p_{2}, \ldots\right)$ and $Q=\left(q_{1}, q_{2}, \ldots\right)$ be sequences of primes. The adding machines $\left(C_{P}, \sigma\right)$ and $\left(C_{Q}, \sigma\right)$ are first return equivalent if there exist clopen subsets $U$ and $V$ of $C_{P}$ and $C_{Q}$, respectively, such that $\left(U, \sigma_{U}\right)$ and $\left(V, \sigma_{V}\right)$ are conjugate, that is, there is a homeomorphism $h: U \rightarrow V$ such that $h \circ \sigma_{U}=\sigma_{V} \circ h$.

\section{THE CLASSIFICATION THEOREM}

Theorem. Let $P=\left(p_{1}, p_{2}, \ldots\right)$ and $Q=\left(q_{1}, q_{2}, \ldots\right)$ be sequences of primes. The following conditions are equivalent.

(a) The spaces $S_{P}$ and $S_{Q}$ are homeomorphic.

(b) The adding machines $\left(C_{P}, \sigma\right)$ and $\left(C_{Q}, \sigma\right)$ are first return equivalent.

(c) $P \sim Q$.

Proof. (a) and (b) are equivalent. This is essentially proved in [7] and a similar proof can be found in [1] and [2].

(b) and (c) are equivalent. First suppose that $P \sim Q$. We may assume that the primes to be deleted are $p_{1}, \ldots, p_{s}$ and $q_{1}, \ldots, q_{t}$, since a change in the order of any finite sequence of primes does not change the isomorphism type of the adding machine. Let $P^{\prime}=\left(p_{s+1}, p_{s+2}, \ldots\right)$ and $Q^{\prime}=\left(q_{t+1}, q_{t+2}, \ldots\right)$ be the derived sequences. We claim that the return maps to $U=\{0\} \times \cdots \times\{0\} \times$ $\prod_{i=s+1}^{\infty} \bar{p}_{i}$ and $V=\{0\} \times \cdots \times\{0\} \times \prod_{i=t+1}^{\infty} \bar{q}_{i}$ are conjugate. In other words, 
$\left(C_{P^{\prime}}, \sigma\right)$ and $\left(C_{Q^{\prime}}, \sigma\right)$ are conjugate. At first the conjugating homeomorphism is defined on one orbit only: let $e=(0,0,0, \ldots)$; then $h\left(\sigma^{n}(e)\right)=\sigma^{n}(e)$. The map $h$ is an isomorphism from $\mathbb{Z}$ with the $P^{\prime}$-adic topology to $\mathbb{Z}$ with the $Q^{\prime}$-adic topology. These subgroups are dense in $C_{P^{\prime}}$ and $C_{Q^{\prime}}$, respectively. Therefore $h$ can be extended to a conjugating isomorphism.

To prove (b) $\rightarrow$ (c), let $U$ and $V$ be clopen subsets of $\left(C_{P}, \sigma\right)$ and $\left(C_{Q}, \sigma\right)$, respectively, and let $h: U \rightarrow V$ be a homeomorphism, conjugating $\sigma_{U}$ and $\sigma_{V}$. Let $x \in U$ and write $y=h(x)$. Without loss of generality we may assume that $U=\left\{x_{1}\right\} \times \cdots \times\left\{x_{k}\right\} \times \prod_{i=k+1}^{\infty} \bar{p}_{i}$. Because of compactness there exists a number $l$ and there are points $y^{1}, \ldots, y^{N}$ such that $V$ is the disjoint union of the sets $\left\{y_{1}^{j}\right\} \times \cdots \times\left\{y_{l}^{j}\right\} \times \prod_{i=l+1}^{\infty} \bar{q}_{i}, j=1, \ldots, N$. It is to be observed that these $N$ sets are permuted by $\sigma_{V}$. Let $m$ be any number greater than $l+1$. By continuity of $h$ there is an $n$ such that

$$
h\left(\left\{x_{1}\right\} \times \cdots \times\left\{x_{n}\right\} \times \prod_{i=n+1}^{\infty} \bar{p}_{i}\right) \subset\left\{y_{1}\right\} \times \cdots \times\left\{y_{m}\right\} \times \prod_{i=m+1}^{\infty} \bar{q}_{i} .
$$

Inspection of the adding machines and their conjugation shows that for some number $M$ we have

$$
p_{k+1} \cdot p_{k+2} \cdots \cdot p_{n}=M \cdot N \cdot q_{l+1} \cdots \cdots q_{m} .
$$

Indeed an element of $U$ returns to $\left\{x_{1}\right\} \times \cdots \times\left\{x_{n}\right\} \times \prod_{i=n+1}^{\infty} \bar{p}_{i}$ precisely once every $p_{k+1} \cdot p_{k+2} \cdots \cdot p_{n}$ times, whereas an element of $V$ returns to $\left\{y_{1}\right\} \times \cdots \times\left\{y_{m}\right\} \times \prod_{i=m+1}^{\infty} \bar{q}_{i}$ precisely once every $N \cdot q_{l+1} \cdots \cdots q_{m}$ times. We conclude that $p_{k+1} \cdots \cdots p_{n}$ must be divisible by $N \cdot q_{l+1} \cdots \cdots q_{m}$. This shows that the tail $\left(q_{l+1}, q_{l+2}, \ldots\right)$ of $Q$ can be mapped injectively in the sequence $P$. Reversing the roles of $P$ and $Q$ we find that some tail of $P$ can be mapped injectively in $Q$. It then easily follows that $P \sim Q$.

\section{REFERENCES}

1. J. M. Aarts, The structure of orbits in dynamical systems, Fund. Math. 129 (1988), 39-58.

2. J. M. Aarts and Z. Frolík, Homeomorphisms of $Q$ and the orbit classification of flows, Rend. Circ. Mat. Palermo (2) 18 (1988), 16-25.

3. R. H. Bing, A simple closed curve is the only homogeneous bounded plane continuum that contains an arc, Canad. J. Math. 12 (1960), 209-230.

4. E. Hewitt and K. A. Ross, Abstract harmonic analysis, vol. I, Springer-Verlag, Berlin, 1963.

5. M. C. McCord, Inverse limit sequences with covering maps, Trans. Amer. Math. Soc. 114 (1965), 197-209.

6. V. V. Nemytskii and V. V. Stepanov, Qualitative theory of differential equations, Princeton Univ. Press, Princeton, NJ, 1960.

7. B. Parry and D. Sullivan, A topological invariant of flows on 1-dimensional spaces, Topology 14 (1975), 297-299.

Delft University of Technology, Delft, The Netherlands 